\title{
Automatic Understanding of Signals
}

\author{
Ryszard Tadeusiewicz \\ Biocybernetic Department of the AGH University of Science and Technology \\ Al. Mickiewicza 30, PL-30-059 Kraków, Poland; rtad@uci.agh.edu.pl
}

\begin{abstract}
Using computers for different task including enhancement, description and evaluation of signals we typically include three steps: signal processing (for obtaining signal of better quality), signal analysis (for extraction and calculation of useful features of the signal), and signal recognition (for classification of signals and for aiding of decision making processes). In paper next step is proposed and presented: automatic understanding of the signal merit content. The idea of Automatic understanding is described on general plane and illustrated using problems taken from the medical imaging problems. As can be shown, automatic understanding of the signals can be used in population investigations (as a filter for preliminary selection patients with problems exacting physicians assistance), or as additional intelligent tool for in depth analysis especially difficult diagnostics problems, as well as new kind of searching machine for content based searching in multimedial databases. In paper is presented general idea of two-directional, knowledge-based data flow when signals must be understood, instead of one-directional sequence of processing step when the signal can be only analyzed and classified.
\end{abstract}

\section{Introduction}

In this paper the new approach to the processing and analysis of signals is proposed [2], [3]. We do try to introduce term (and methodology) of signal understanding, as a new step in the way starting form signal processing, followed by analysis and classification (recognition). The general view of the situation of new technology under consideration in context of much more known techniques of signal processing, analysis, segmentation and classification is shown bellow (Fig.1). The idea of signal understanding can be applied for all signals [8], [9], because methodology under consideration is general and useful in different situations, but majority of explanations given bellow is related to the images, because man can imagine complex situations usually on the base of pictorial presentations [1], [4], [7]. Also concrete examples of the applications of the automatic understanding will be connected with images, especially medical ones. It takes from the fact, that we do need new method of analysis exactly for medical images, because this type of images are especially difficult for traditional analysis and recognition (Fig.2).

The most detail analysis of this problem (suing automatic understanding methodology for medical images) will be done in the paper by prof. Marek Ogiela, also published in this book. We must here show general view of this methodology, starting from very known basis of typical signal processing, analysis, and recognition. 


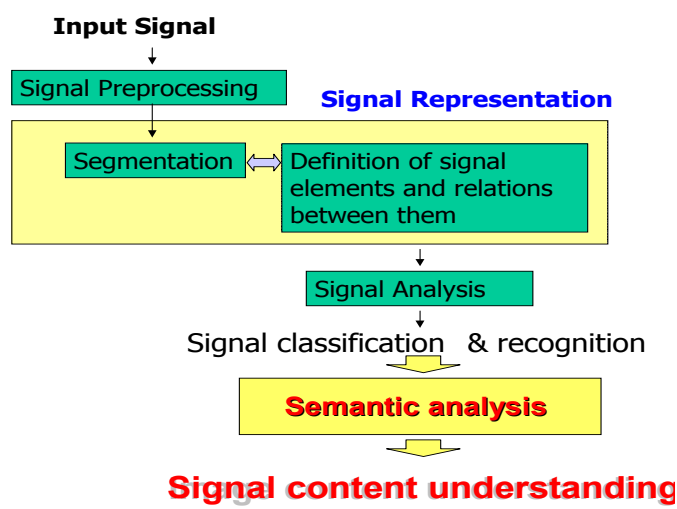

Fig. 1. Localization of signal understanding

Lets compare two examples of ERCP images of pancreatic duct with pancreatits (permanent inflammation)

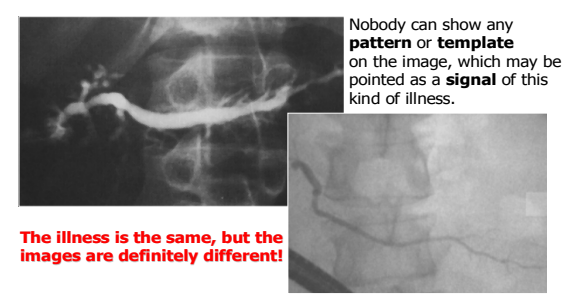

The same situation we can find when analyzing of ERCP images presented pancreatic cancer.

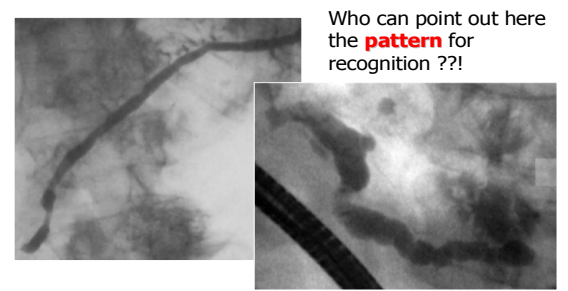

Fig. 2. Difficulties with recognition of medical images force us to develop new methodology: automatic understanding of the images

\section{Signal processing, analysis, recognition, and understanding}

Thanks to many engineers' inventiveness and their attempts to find still better ways to collect and present different signals we have now very useful sequence of signal elaboration and evaluation [5], [6]. This sequence includes:

- signal processing - aimed to improve the quality of the signal (filtering, enhancement, deconvolution, sharpening and contrasting etc.) and to segmentation of the continuous signal into parts for taking into future consideration only those parts (objects) which are vital from the application point of view

- signal analysis - aimed to determine the features of the whole signal or specified objects, and compute the value of quantitative parameters (for example spectral or space-temporal) of the signal samples

- signal recognition (or pattern recognition) - aimed to identify and classify the highlighted elements of the signal through indexing them as objects 
belonging to certain categories determined a priori- mainly on the basis of their features.

Relations between mentioned steps of signal evaluation are shown on Fig.3.

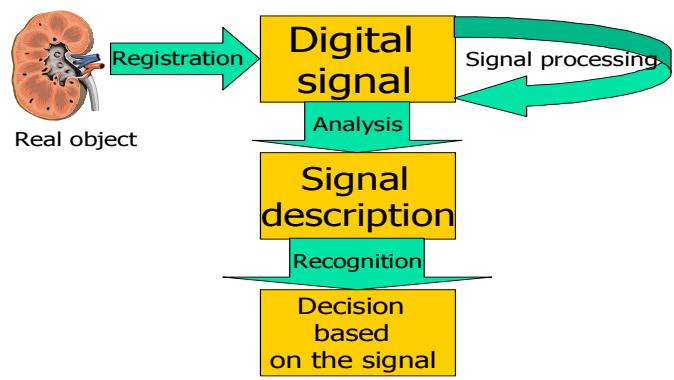

Fig. 3. Interrelations between classical components of signal evaluation

All aforementioned techniques of computer processing, analysis and recognition of the signals prove insufficient to tackle all the tasks and problems that arise in connection with the use - for example - images as valuable sources of medical information. The most fundamental and vital aspect is interpretation of collected data, referring to the physician's knowledge. At the present stage the physicians' knowledge of correct and pathological anatomy and physiology of examined organs and structures is the only key to their proper understanding why medical image looks the way it does. Owing to computer processing of images the shapes of organs and lesions can be better visible; however the physicians provide their interpretation of the image. Dependencies and relationships between "traditional triad" of signal processing techniques and the new approach given by means of automatic understanding of the signals are summarized in Table 1 on the base

Table 1. Dependencies and relationships between "traditional triad" of signal processing techniques and the new approach

\begin{tabular}{|c|c|c|}
\hline $\begin{array}{c}\text { Stage of image } \\
\text { utilization }\end{array}$ & $\begin{array}{c}\text { Question answered in } \\
\text { traditional approach }\end{array}$ & $\begin{array}{c}\text { Question answered in new } \\
\text { (proposed) approach }\end{array}$ \\
\hline $\begin{array}{c}\text { Image } \\
\text { processing }\end{array}$ & $\begin{array}{c}\text { How to increase quality and } \\
\text { visibility of the image? }\end{array}$ & $\begin{array}{c}\text { What follows the } \\
\text { visualized details? }\end{array}$ \\
\hline $\begin{array}{c}\text { Image } \\
\text { analysis }\end{array}$ & $\begin{array}{c}\text { Which are the exact } \\
\text { values of selected } \\
\text { features of the image? }\end{array}$ & $\begin{array}{c}\text { What is the meaning } \\
\text { of the features } \\
\text { extracted from the image? }\end{array}$ \\
\hline $\begin{array}{c}\text { Image } \\
\text { recognition }\end{array}$ & $\begin{array}{c}\text { To which classes } \\
\text { (patterns) do the } \\
\text { selected objects on the } \\
\text { image belongs? }\end{array}$ & $\begin{array}{c}\text { What are the results } \\
\text { of the fact, that some } \\
\text { objects belongs to } \\
\text { particular classes? }\end{array}$ \\
\hline
\end{tabular}


of images processing versus understanding. Technically speaking, the difference between signal recognition and signal understanding includes following assumptions:

- in case of recognition we always have a fixed number of a priori known classes and the task demands only extraction of all these features of the signal, which are necessary and sufficient for differentiation between classes under consideration. After signal processing and analysis we do obtain the number (or name) of proper class, but without deeper analysis of signal meaning;

- in case of understanding we have not any a priori known classes or templates, because in fact the potential number of possible classes of signals goes to infinity. So after processing we do obtain a description of the signal content without using any a priori known classification, because even the criteria of classification are constructed and developed during the automatic reasoning process.

\section{Areas of applications for automatic understanding of signals}

The technique of signal understanding have several very interesting applications. Now, we will indicate and analyze three types of $\mathbf{T}$-formed areas of its applications, especially in medicine (Fig.4). The upper part of the considered

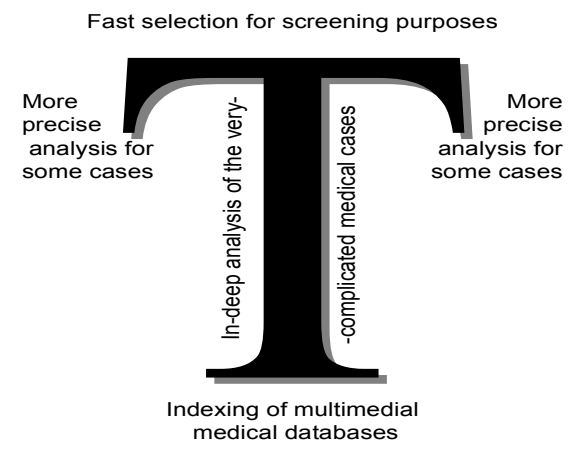

Fig. 4. Symbolic structure and abbreviated characteristics of areas of medical application for automatic understanding of signal

diagram consists of population investigation area. In this type of application tasks of a computer capable of signal understanding require the analysis of a large number of data - this area of applications is symbolized with the top dash of the letter $T$, because the range of analysis is wide, and the depth of analysis is rather small. In certain cases we may perform a deeper analysis, as will be shown in the drawing and discussed below. 
The other area of application of automatic signal understanding technique is deep and requires a detailed analysis of particularly difficult problems, especially in case of doubts and difficulties in deciding on the final diagnosis. In such cases, a computer well equipped with automatic signal understanding programs may support a diagnostician analyzing subtle and hardly visible features to improve conformity of the diagnosis (calculated as the average of many examined cases). Certainly, such ambitious target may be reached only in relation to a narrow area of problems, hence the symbolic structure of application of automatic image understanding methods illustrated in Fig.4 reflects the discussed problems with a part of the letter $\mathrm{T}$ characterized with a very small width and at the same time going deep into the problems under consideration.

Furthermore, the more detailed area of problems connected with a part of the T letter, usually called a "sheriff" located at the base of the letter symbolizes opportunities connected with application of the automatic signal understanding to semantic indexation (oriented to the meaning not to the form!) of multimedial medical data bases. This problem requires more extensive introduction for better understanding and will be further discussed below. Here, we only note that one of the furthest- reaching advantages of the automatic signal understanding is possibility of overcoming a problem of finding meritoriously (and not only formally) adequate records in multimedia medical data bases. Automatic signal understanding technique will allow the users to search for records in Internet (and also in other image and multimedia resources) based on meritorious criteria (i.e. related to similar types of pathology) and not on superficial similarity of signals. Automatic signal understanding will allow automatic indexation of numerous medical data bases which are extremely difficult to search (because they are so numerous!) and that the same technique may significantly facilitate automatic asking questioning of information systems and obtaining required information with a very narrow margin of wrong or inadequate answers.

Having described the above general application of automatic image understanding technique we will now try to characterize it in the following subsections. We will concentrate mainly on what we need, not what we may reach, because this part of the book is devoted to asking questions, and only further chapters of the book will be devoted to solving the problems.

\subsection{Automatic understanding of the signals as a tool for preliminary classification of screening results}

The first area of application of automatic signal understanding is a computer tool for preliminary analysis and selection of a large number of diagnostic signals (for example images or ECG registrations) obtained during population investigations [3], [8]. This refers to the upper part of the already mentioned " $T$ " letter. The role of automatic signal understanding in the cases is concentrated on sensible detection and indication of all the signals (images) contain- 
ing disturbing symptoms in patients whose cases require thorough analysis. The role of an intelligent computer program is that of a "preliminary filter" extracting a small number interesting cases hidden in the ocean of trivial cases, and may be extremely useful in screening of large populations (Fig.5).

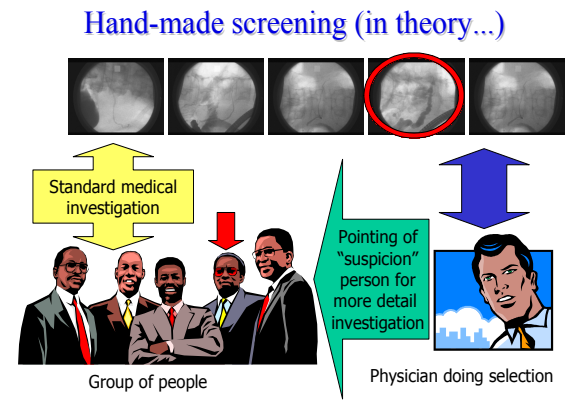

Fig. 5. Theoretical model of "ideal hand made" screening
Hand-made screening (in practice...)

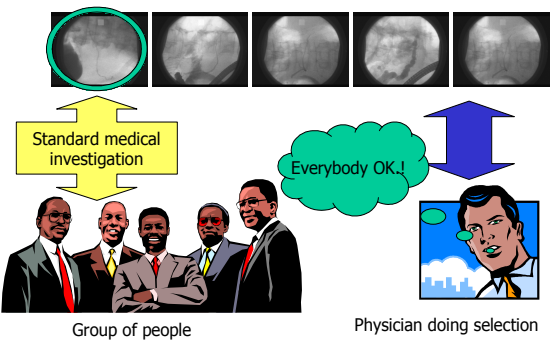

Fig. 6. Mistakes in "real hand made" screening

However, analyzing such a large collection of diagnostic materials is a real bottleneck. Focusing only on medical images, we may state, that even the best qualified and hard-working practitioner is able to analyze less than a hundred images an hour. What is more, the majority of such images would illustrate cases with no pathological features, and a doctor might get bored and deconcentrated, due to specifically understood mental, though not sensoric, deprivation. Consequently, when an image containing early not visible at first sight but noticeable symptoms of a disease appear, it is possible that a doctor will treat them as images of a healthy person - usually it will end with fatal consequences for a patient whose disease has not been spotted (Fig.6). Such incident may put the whole idea of screening in question.

In this situation we may and we should employ the modern computer technique, especially the automatic image understanding technique described in this paper (Fig.7). Although it is well known we want to say again that automatic image analysis is always carried out with the same precision and that a well constructed analyzing program will always detect pathological symptoms, even the frailest ones, regardless of how many boring trivial images it has had to analyze before. This feature is worth striving for!

The most important task of the computer visual system is the preselection of registered images (Fig.7 left) because it is extremely difficult for human beings to extract important diagnostic information from numerous and diversified images, even when we do not take into consideration a large number of images showing tissues and structures free from any pathological symptoms. After selecting "suspicion" data automatic system can also help to the physician in detail analysis of details of the image under considera- 


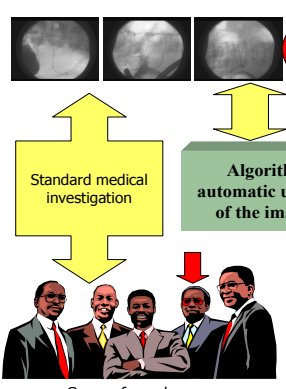

Group of people

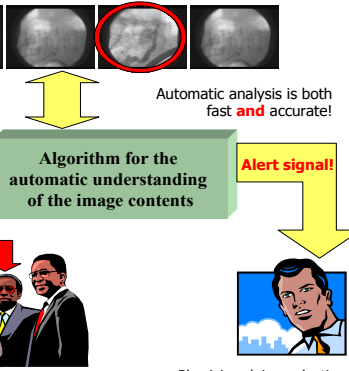

Physician doing selectio

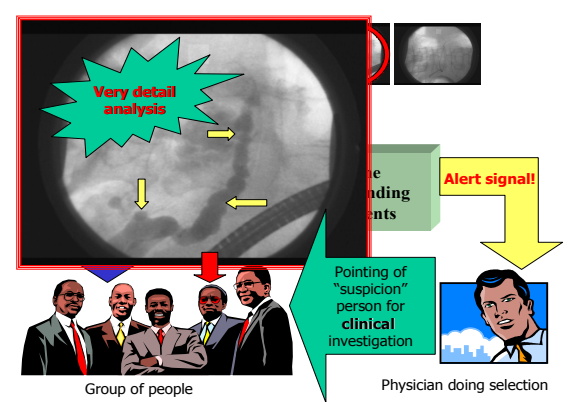

Fig. 7. Automatic system helping to the physician in screening job (selection of "suspicion" data and computer aided its analysis)

tion (Fig.7 right) - but this is in fact not so important part of this job. The most important goal for automatic understanding system is connected with generating of "alert signals" in this area of applications.

\subsection{Automatic understanding in difficult medical problems}

The above described task of employing automatic understanding medical images only for preselection of "suspicious" images in screening results [4], [5], [6]. We cannot expect more with regard to this task at the present stage of development in information technology. The risk of assigning the final decision making to the computer would be too risky in case of screening because of a very large number of possible pathologies, so in this case, medical staff must bear the entire responsibility for the decision making process, and we cannot do much about it.

If we start with narrowing the application field of the created algorithm of automatic image understanding to selected specialist problems (even very difficult ones) we will have different situation, which is much more interesting from the scientific research point of view. If we locate the considered computer system in a clinical hospital (i.e. cardiology or gastroenterology clinics) we know the field of its operation, because patients admitted to the clinic have been already diagnosed and they are seriously ill. Building a computer system designed for intelligent image analysis for the needs of the defined clinic we may take two assumptions, which will significantly narrow the task and consequently allow us to have the analyzing process more advanced (Fig.8). Hence, the "leg" of $\mathrm{T}$ is so narrow and long. On the other hand however, these are usually the most difficult cases (also in diagnostic sense), because simple cases are treated in ordinary hospitals, and not in clinics. Therefore it is necessary to perform thorough analysis of all the data concerning a patient, including medical images, because usually diagnosis must be performed in atypical situation, or several probable diagnostic hypotheses must be taken into account. Because clinical problems require a thorough analysis, they 

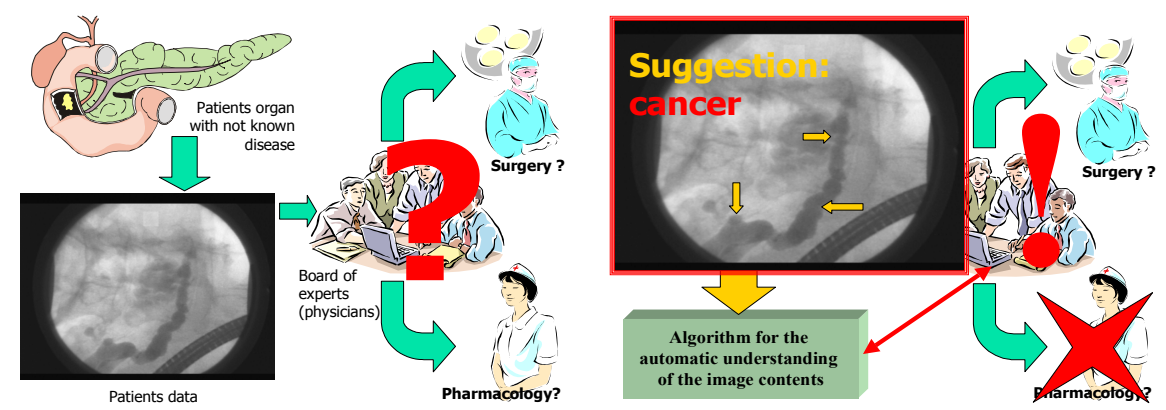

Fig. 8. Assistance of the "automatic image understanding system" in difficult diagnosis problem

are symbolized by this part of $\mathrm{T}$ which reaches deep down to fundamental decisions and appraisals based on the very careful analysis of very small, hidden or hardly detectable features of the images (Fig.8).

\subsection{Automatic understanding of the images as a tool for semantic searching in databases and successful web crawling}

A tool described in this paper consisting of a set of automatic signal understanding methods may have one more application symbolized by the bottom bar of T letter (see Fig.4). The characteristic feature of the signals (registration of various information not semantically reducible to a text or a set of digits, especially medical images) is that they are usually very difficult from the point of view of intelligent service, as their ontologies have not been well developed [7].

However, multimedia resources collected in the numerical form are still growing. Difficulties in usage of such multimedia data may be considered from various points of view, but we shall focus on searching techniques with special consideration of semantic searching oriented to the meritorious content. Semantic searching is somehow inconvenient, because it may be very difficult to find multimedia information meeting the defined and meritorious criteria not supported with precise (and properly made!) verbal description, but based only on the registered signal itself (i.e. image bitmap). Now, we may signal the next application of the technique of automatic image understanding described in this book treated as a tool for intelligent indexation.

Let us imagine that resources of multimedial signals collected in digital carriers do not have the detailed semantic description. For example hospital databases known to the authors, as well as enormous and still growing resources of medical images collected and accessible on websites lack such descriptions. It makes finding an image illustrating a chosen problem (i.e. one needed for illustration of didactic argument) extremely difficult, and in extreme cases it may mean that we have to review very many images (spending 
a lot of money and time on getting them via Internet) until we find the right one. The situation is even more difficult when we want to see all available images with the same meritorious content (i.e. data of all the patients with identically located and similarly advanced cancer as in the patient being treated at present). Such needs are quite frequent, because the method of referring to therapy applied to other patients is very popular in the process of choosing the therapy for a given patient. However, the problem of meritorious content related searching of multimedia databases has not been solved yet.

Concept of automatic image understanding discussed in this paper creates interesting possibilities. Having a tool not only analyzing but also semantically describing an image, we can follow the following model of procedure.

A computer equipped with an automatic image understanding program systematically views accessible multimedia medical databases and tries "to understand" the content of each image. Certainly, this should be a background task performed only in time when there are no perpetual commendations of the user. Upon such review of certain images located in databases it will be possible to create their semantic descriptions automatically (their automatically analyzed content will be understandable for a computer). When the semantic meaning of an automatically analyzed image is found out, a record identifier containing such a "decoded" image will be linked to the record containing the whole "deducted" semantic description of the image (Fig.9).

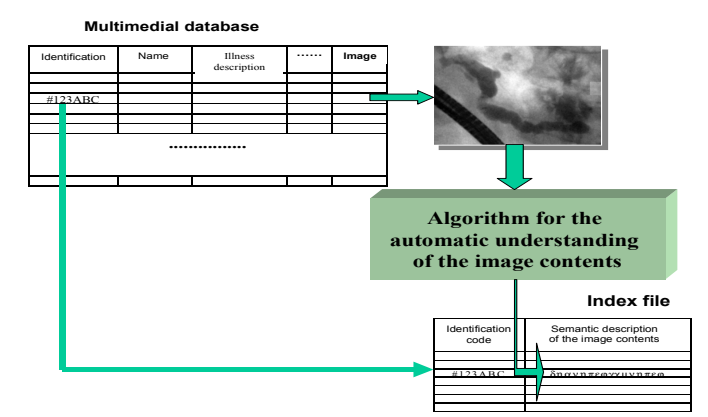

Fig. 9. Automatic image understanding in the process of multimedia database indexation

This description usually consists of a series of symbols denoting the semantic content of the image, usually illegible and incomprehensible for a human (that is why they are presented in drawings as Greek letters). Principles of creating such descriptions and methods of operation will be described in the further part of the book. It may only be noticed here, that it will require a technique based on the methods of mathematical linguistics using specifically constructed graph grammars. 
An automatically generated semantic description should be located in a special index file of a computer, which scans the base. The description should be always located outside the base in order not "clutter" the original database (automatically generated semantic descriptions of an image may contain errors!), and searching of separate index files is much more faster than searching the whole base expanded with large volume digital image records. A relevant record should appear in the index file for each image with the code representing its meaning settled in result of the automatic analysis. A number of images understandable for a computer vary, and probably, for a very long time, we will not be able to create semantic indexation mechanism able to refer automatically the adequate description to each medical image in any database. However, taking into account designation of the whole process, this defect is not very significant.

Naturally, all the images with distinct legible features will be the first to be indexed efficiently. In case of such images semantic analysis will be successful and consequently they will be unambiguously classified with the automatic indexation procedure. Searching process applied in this connection will find such "clear-cut" cases first. Taking into account the purpose of searching, it cannot be considered as a defect. We must remember that semantic searching database for medical images usually is focused on finding images that may serve as didactic examples or reference objects in the process of finding the optimal diagnosis - here the usefulness of "good" images seems to be indisputable. In this respect, finding the most distinct and the most typical images, neglecting ambiguous or highly atypical cases should be consider not as a drawback but as an advantage of the automatic image indexation procedure. The only problem (if any) is that not all the objects of the database will be indexed in result of such indexation. Namely, some objects, too difficult for the automatic understanding procedure, will not have an automatically settled semantic description of their content. In result, they will be of no use for automatic reasoning process. However, if such a database is rich enough (the technique considered here is introduced because databases have grown very large), the process of automatic indexation will provide us with quite a large number of identified (usable) records that we will not face the problem of deficit.

Let us show now, how to make use of a semantically indexed multimedia database in case we need information on the required subject. We assume that a question to the database has a form of an image ("show me something like this....").The sequence of operations is presented in Fig.10. First, an image of the object we are interested in (which constitutes the basic part of our request) is subjected to the automatic procedure setting its meritorious content, same as the procedure used for indexation of the base. A code (analogical to the code used for base indexation) created in result of the attempted automatic understanding of the meritorious content of the image allows finding all these positions in the index file that show the defined 
degree of similarity to the code of the model image included in the request. When identifiers of medical records corresponding to these positions in the index file in the indexed databases are known, it is possible to extract all the required information and use it according to the purpose. Final effect can be (and ought to be!) optimal selection of proper therapy - Fig.11.

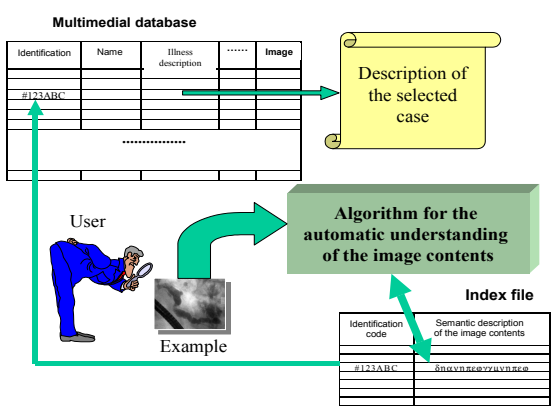

Fig. 10. Context related searching for required information in the semantically indexed multimedia database

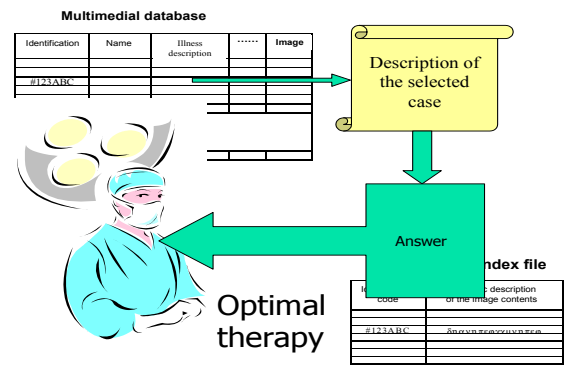

Fig. 11. Selecting of the optimal therapy using multimedial medical database and intelligent (based on images understanding) searching machine

The above-described mechanism of indexation and semantic searching the required medical images may be organized according to two patterns. The first pattern is designed for a client of the database. In this pattern, indexation of objects in databases and conversions of requests sent to databases in the semantic form described in this book take place in the client's computer. In other words, users who want semantic-oriented access to information resources, request the computer to carry out the indexation of relevant databases. Indexation is run according to the pattern shown in Fig.9, however, it must be taken under consideration that the database is located at a server (sometimes very distant one), and the tool for image intelligent understanding and the index file created by this tool are in the client's computer. The strong point of this approach is that any user having access to the scanned database and a tool for automatic understanding of the image content may realize it at any time. The weak point is the high cost of indexation resulted from the fact that practically each image contained in database must be transmitted via the web to a client's computer, which will try to understand the content In case many clients want to use the same server, the same (arduous!) job will be unnecessarily repeated many times.

Indexation performed by the owner of the database is a complementary solution. In this case automatic image understanding program must be located both in a server (for continual indexation of the database, which, of course, keeps changing its content all the time) and in the client's computer (to convert images displayed during requests). 


\section{General description of the fundamental ideas of automatic understanding of the signals}

Very important difference between all traditional ways of automatic signal processing (or recognition) and the new paradigm of signal understanding includes one directional scheme of the data flow in traditional methods and definitely two -directional interaction between features extracted from the signal analysis and demands taken from the merit knowledge about the signals, given by experts (physicians). Lets se to the Fig.12, on which we can se traditional scheme of the signal (in presented case - image) processing for the medical purpose.

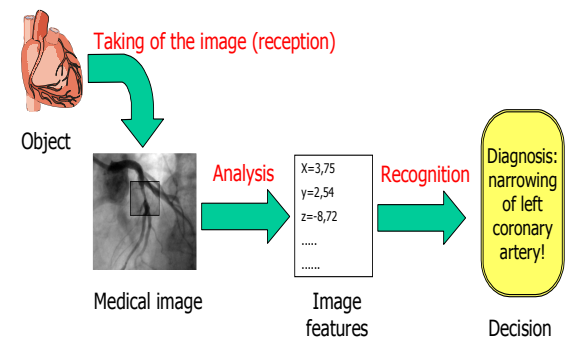

Fig. 12. Traditional method of medical images recognition

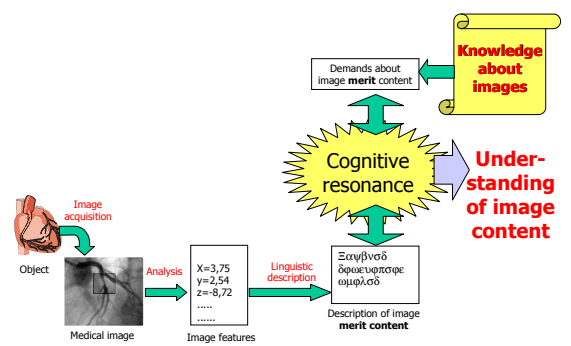

Fig. 13. The main scheme of image understanding

In contrast to this simple scheme given for classical recognition - during signal understanding we always have two-directional flow of information (Fig.13).

On both above presented figures we can se, that when we use traditional pattern recognition paradigm, all process of the signal (image) analysis is based on feed-forward (one-direction flow of signals) scheme. In contrast, when we use automatic understanding of the images total input data stream (all features obtained by means of analysis of the image under consideration) must be compared with the stream of demands generated by dedicated source of knowledge - Fig.13. The demands are always connected with particular (selected) hypothesis of semantic interpretation of the image content. So we can stress, that proposed "demands" are kind of postulates, describing (on the base of merit knowledge about the image) desired values of some (selected) features of the image. The selected parameters of the image under consideration must have desired values when some assumption about semantic interpretation of the image content can be validated as true. When the parameters of the input image are different - it can be interpreted as partial falsification of the one of possible hypothesis about the merit content of the image - but still can not be considered as a final solution. 
Because of this specific model of inference we called our mechanism "cognitive resonance". This name is proper to our ideas, because during comparison process between features calculated for input image and demands generated by the source of knowledge - we can observe amplification of some hypothesis (about merit meaning of image content) when other (competitive) hypothesis become weeks. It is very similar to the interferential image formed during mutual activity of two sources of waves: in some points of the space waves can gain one other, in other points there are opposite phases and final result is near zero.

Such structure of the system for image understanding corresponds with one of the very known models of natural visual perception by man, named knowledge based perception. Humans eye can not recognize object if the brain have not a template for such view. It remains true although the brain knows the object, but in another view, what means another signals coming to the visual cortex.

The description of the cognitive resonance presented here is of course the most simplified one. In fact the set of methods and formulas used by real parser designed by us especially for this works is much, much more complicated! But it will be shown on the base of practical examples in paper by prof. Marek Ogiela.

\section{Conclusions}

New idea of automatic understanding of signals can be found as next logical and very natural step after very known levels of signal processing, signal analysis and signal recognition. Idea under consideration is based on the assumption, that in many signals one can discover besides its form (suitable for processing, analysis and recognition) also its merit sense (semantic content), which is much more interesting in many applications. Contemporary computers can perform many signal processing tasks. It is now comfortable and easy, because algorithms (and ready for use programs) for such processing are very known. So if we know, what we wont to do, we can use a lot of specialized methods e.g. FFT transform, JPEG and MPEG compression, blind deconvolution, discrimination analysis, pattern recognition, neural network application etc. We can also perform this kind of signal processing tasks even faster and faster, because of progress in DSP technology.

But being equipped with all this methods, algorithms, and processors we very often can not solve practical problems, because the useful information is hidden in semantic content, which must be discovered by means of understanding of the signal instead of simple classification or recognition. The proposition given in this paper was explored previously for understanding of medical images, ad it is why many examples in the papers body was connected with such case of images. But the idea of automatic understanding if signals is much more general and should be analyzed on the base of different 
types of signals. The goal of this work is to encourage researchers for trying this new approach in the variety of problems.

\section{Acknowledgment}

The AGH University of Science and Technology supported this work under Grant No.11.11.120.249.

\section{References}

1. Kurgan, L.A., Cios, K.J., Tadeusiewicz, R., Ogiela, M. (2001) Goodenday L.S., Knowledge Discovery Approach to Automated Cardiac SPECT Diagnosis. Artificial Intelligence in Medicine. 23 (2), 149-189

2. Leś, Z., Tadeusiewicz, R. (2000) Shape Understanding System - Generating Exemplars of the Polygon Class. Hamza M.H., Sarfraz E. (eds.): Computer Graphics and Imaging. IASTED/ACTA Press. Anaheim. Calgary, Zurich, 139144

3. Ogiela, M., Tadeusiewicz, R. (2000) Artificial Intelligence Methods in Shape Feature Analysis of Selected Organs in Medical Images. Image Processing \& Communications, 6 (1-2), 3-11

4. Ogiela, M. R., Tadeusiewicz, R. (2001) Image Understanding Methods in Biomedical Informatics and Digital Imaging. Journal of Biomedical Informatics, 34 (6), 377-386

5. Ogiela, M. R., Tadeusiewicz, R. (2002) Syntactic reasoning and pattern recognition for analysis of coronary artery images. Artificial Intelligence in Medicine, 26, 145-159

6. Ogiela, M.R., Tadeusiewicz, R. (2003) Artificial Intelligence Structural Imaging Techniques in Visual Pattern Analysis and Medical Data Understanding. Pattern Recognition, Elsevier 2003, 36/10, 2441-2452

7. Tadeusiewicz, R., Ogiela, M.R. (2003) Artificial Intelligence Techniques in Retrieval of Visual Data Semantic Information. Menasalvas E., Segovia J., Szczepaniak P.S. (eds.): Advances in Web Intelligence. Lecture Notes in Artificial Intelligence, No. 2663, Springer Verlag, 18-27

8. Tadeusiewicz, R., Ogiela, M.R. (2003) Machine Perception and Automatic Understanding of Medical Visualizations. Damczyk M. (ed.): Automatic Image Processing in Production Process. Second Polish-German Seminar, CAMT, Wrocław 2003, 39-48

9. Wszołek, W., Tadeusiewicz, R., Izworski, A., Wszołek, T. (2002) Recognition and Understanding of the Pathological Speech Using Artificial Intelligence Methods. Hamza M.H. (ed.): Artificial Intelligence and Applications, IASTED, ACTA Press Anaheim-Calgary-Zurich, 504-508. 